\title{
Atuação do profissional de saúde mental nos núcleos de apoio à saúde da família: Uma revisão integrativa
}

\section{Professional performance in mental health in the family health support centers: An integrative review}

\author{
Shirley Christina Melo Araújo \\ Fisioterapeuta e Mestre em Saúde Pública pelo Centro de Pesquisa Aggeu Magalhães da Fundação Oswaldo Cruz. E- \\ mail: shirleycmaraujo@ hotmail.com \\ Mayara Leite Alves da Silva \\ Graduanda em Fisioterapia pela Universidade Estadual de Ciências da Saúde de Alagoas. E-mail: \\ mayaraifal@gmail.com \\ Maykon Wanderley Leite Alves da Silva \\ Graduando em Medicina pela Universidade Estadual de Ciências da Saúde de Alagoas. E-mail: \\ maykonwanderleyleite@gmail.com
}

\begin{abstract}
Resumo: A saúde mental depende da qualidade de vida cognitiva e emocional de cada indivíduo. Os desequilíbrios mentais são condições que afetam significativamente o comportamento humano e o convívio social e, nesse contexto, faz-se necessário um olhar humanizado dos profissionais pertencentes aos Núcleos de Apoio à Saúde da Família (NASF). Diante disso, surgiu o seguinte questionamento: qual seria a atuação dos profissionais de saúde mental na atenção básica? O objetivo do estudo foi realizar um levantamento das publicações sobre a atuação dos profissionais nos NASFs na área de saúde mental durante o período de 2012 a 2017. Trata-se de uma revisão integrativa da literatura realizada nas bases de dados do SciELO, do LILACS e do PubMed, com a utilização dos descritores "Mental Health" and "Primary Attention" and "Family Health Support Center". Os critérios de inclusão foram artigos relacionados à temática, publicados na íntegra, no idioma Inglês e Português, durante o período de janeiro de 2012 a janeiro de 2017, ao passo que os critérios de exclusão foram estudos de revisão de literatura, publicações repetidas, resenhas, artigos de opinião e relato de experiência. Nesse sentido, os resultados mostraram que ainda há profissionais da Estratégia Saúde da Família (ESF), assim como no NASF, com dificuldades na abordagem aos pacientes que apresenta distúrbio mental por falta de capacitação e elegem pensamentos biomédicos e hospitalocêntricos. Porém, outra parcela dos trabalhadores relata insegurança quanto ao cuidado e encaminham os pacientes aos Centros de Atenção Psicossocial (CAPS), por ser um ponto de atenção à saúde. Assim, é necessário o matriciamento ofertado às equipes multiprofissionais de saúde mental com o intento de ampliar as ações de saúde e fomentar a humanização da assistência para que seja holística e direcionada.
\end{abstract}

Palavras-chave: Saúde Pública; Serviços de Saúde; Atenção Primária; Apoio Matricial.

\begin{abstract}
Mental health depends on each individual's cognitive and emotional quality of life. Mental imbalances are conditions that significantly affect human behavior and social interaction, and in this context, it is necessary to have a humanized view of the professionals belonging to the Family Health Support Centers. In view of this, the following question was raised: what would be the performance of mental health professionals in primary care? The objective of the study was to carry out a survey of the publications on the performance of professionals in the NASFs in the area of mental health during the period from 2012 to 2017. This is an integrative review of the literature performed in the databases of SciELO, LILACS and of PubMed, using the descriptors "Mental Health" and "Primary Attention" and "Family Health Support Center". The inclusion criteria were articles related to the theme, published in both English and Portuguese, during the period between January 2012 to January 2017, while the exclusion criteria were studies of literature review, repeated publications, reviews, opinion articles and experience reports. In this sense, the results showed that there are still professionals in the Family Health Strategy (FHS), as well as in the NASF, with difficulties in approaching patients who present mental disorder due to lack of qualification and elect biomedical and hospitalocentric thoughts. However, another portion of the assistants report insecurity about care, but they refer patients to the Psychosocial Care Centers (CAPS), because it is a strategic point of the health care network. Thus, it is necessary the matriciamento offered to multiprofessional teams of mental health with the intent to expand health actions and promote the humanization of care so that it is holistic and directed.
\end{abstract}

Keywords: Public health; Health services; Primary attention; Matrix Support. 


\section{INTRODUÇÃO}

A Saúde Mental é mais do que a ausência de doença. Ela está intimamente ligada à saúde física e social de cada indivíduo, uma vez que proporciona o equilíbrio emocional nos conflitos e nas adversidades internas e externas (BRASIL, 2013). Nessa direção, os transtornos mentais são condições que atingem o humor, o raciocínio e o comportamento do paciente. Isso gera consequências como isolamento social. Esse fenômeno pode ser percebido em muitos países (WHO, 2015).

A Reforma Psiquiátrica brasileira teve início no período de crise do modelo assistencial hospitalocêntrico, por volta da década de 70 , no pós reforma sanitária. A partir disso, o Movimento dos Trabalhadores em Saúde Mental (MTSM) denunciou casos de violência nos manicômios, além das péssimas condições de alimentação. Reverberou, nesse período, a luta antimanicomial em prol do fechamento desses hospitais e melhorias na acessibilidade dos serviços de saúde (BRASIL, 2005).

Segundo a Organização Mundial da Saúde, a falta de sanidade mental atinge cerca de 700 milhões de indivíduos no planeta, o que representa $13 \%$ do total de doentes no mundo e, aproximadamente, $70 \%$ dessas pessoas não possuem acesso ao tratamento adequado (OMS, 2008). A partir da magnitude desses índices, o Ministério da Saúde cria os Núcleos de Apoio à Saúde da Família (NASF), por meio da Portaria GM n.154, de 24 de janeiro de 2008, que objetiva apoiar a Estratégia Saúde da Família (ESF) nos serviços de cuidado integral à saúde através de ações interdisciplinares na atenção básica e, com isso, é recomendado que cada NASF disponha de um profissional qualificado na área de saúde mental para melhor acompanhar esses pacientes (BRASIL, 2008).

A equipe multiprofissional do NASF atua nas áreas de atividade física, alimentação, reabilitação, serviço social, saúde mental, saúde da mulher, saúde da criança, homeopatia, acupuntura e assistência farmacêutica a fim de oferecer uma melhor assistência do serviço aos usuários do Sistema Único de Saúde - SUS (BRASIL, 2008).

No Brasil, há 1.705 municípios com população abaixo de 15 mil habitantes com escassas equipes do (NASF), o que promove um atendimento precário aos pacientes com algum distúrbio ou transtorno mental (BRASIL, 2015).

Nesse contexto, se faz necessário um olhar diferenciado na articulação de redes de saúde e, sobretudo, na atenção primária. Com isso, as equipes de Saúde da Família necessitam da integração aos outros profissionais e aos serviços de saúde, relacionados ao bem-estar supracitado dos indivíduos, em busca de prevenção e de promoção da saúde (BRASIL, 2013).

A atenção básica, como direciona a Política Nacional de Atenção Básica (PNAB), deve constituir o primeiro acesso das pessoas ao sistema de saúde, inclusive para aquelas que demandam um cuidado em saúde mental, a partir do oferecimento de uma abordagem integral, que integra o respeito às particularidades à promoção da qualidade de vida das pessoas envolvidas - pacientes e cuidadores (BRASIL, 2013).
Ademais, o engajamento da equipe do NASF com práticas interdisciplinares na saúde mental possui resultados bem-sucedidos, já que os profissionais são capazes de entender a realidade dos usuários, consolidando a efetividade dos princípios do SUS, com a integralidade e a resolubilidade dos problemas, fundamentais para o exercício profissional que impacte positivamente na vida dos pacientes (FIGUEIREDO; CAMPOS, 2008). Espera-se dos profissionais que atuam nos NASFs a adoção de um processo de trabalho, que haja escuta qualificada das queixas dos pacientes e a capacidade de os orientar de forma adequada. Isso favorecerá uma atenção humanizada com reabilitação psicossocial, além de oferecer um suporte aos familiares envolvidos (CORREIA et al., 2011).

Estudos tem mostrado que esse suporte dos familiares, também chamados de cuidadores primários, torna-se importante, uma vez que na maioria dos casos se sentem sobrecarregados (ORUCHE et al., 2015).

Além disso, o distúrbio mental interfere não somente no convívio e na sobrecarga à família envolvida, mas também na educação e na inserção desses indivíduos no mercado de trabalho, o que influencia negativamente o desenvolvimento pessoal e profissional (ERVIN et al., 2014).

Neste sentido, o NASF mostra-se como possibilitador de uma rede mais integrada nos cuidados básicos de saúde, já que permite identificar as fragilidades dos doentes mentais e reinseri-los na sociedade, assim como maior aproximação da família e dos profissionais da área da saúde, por intermédio da desinstitucionalização, assistência holística e desconstrução de conceitos sobre a loucura, arraigadas na lógica sanitária hegemônica (SCHUTEL et al., 2015).

Por acreditar que o conhecimento e a orientação das políticas de atenção à saúde mental são de fundamental importância não somente para os gestores, mas também para os prestadores de serviços, familiares e os próprios usuários, cabe, portanto, a eles o protagonismo no processo de reabilitação em saúde mental e na valorizarização das singularidades dos sujeitos com melhorias nas condições de acesso e de qualidade da assistencial.

Diante disso, surgiu o seguinte questionamento: qual seria a atuação dos profissionais de saúde mental na atenção básica?

Este trabalho tem como objetivo realizar um levantamento bibliográfico dos principais temas acerca da atuação do profissional de saúde mental nos NASFs.

\section{MATERIAL E MÉTODOS}

Trata-se de uma revisão integrativa da literatura que consiste na elaboração de uma ampla análise literária e na promoção da discussão referente à atuação do profissional nos Núcleos de Apoio à Saúde da Família (NASF) no tocante à saúde mental. Esse método de pesquisa caracteriza-se por sintetizar o conhecimento científico e analisar as produções sobre o tema proposto, além de levantar possíveis lacunas do conhecimento que precisam ser sanadas através de novos estudos (KAKUSHI; ÉVORA, 2016).

Para tanto, a revisão integrativa utiliza-se da Prática Baseada em Evidência (PBE) apresentando-se etapas para 
sua execução, nas quais prioriza a elaboração da pergunta norteadora, busca na literatura, coleta de dados, avaliação dos estudos incluídos, interpretação dos resultados e apresentação da revisão integrativa (DE SOUZA et al., 2010).

O acesso às publicações ocorreu em junho, julho e agosto de 2017, nas bases de dados SciELO, LILACS e PubMed. Desse modo, foi utilizada uma busca avançada, a partir do cruzamento dos seguintes descritores: "Mental Helth" And "Primary Attention" And "Family Health Support Center" e seus respectivos descritores em português. Assim, uma análise descritiva dos estudos foi realizada.

Os critérios de inclusão foram artigos relacionados à temática, publicados na íntegra, no idioma Inglês e Português, durante o período de janeiro de 2012 a janeiro de 2017, ao passo que os critérios de exclusão foram: revisão da literatura, publicações repetidas, resenhas, artigos de opinião e relato de experiência.

Nesse prisma, foram extraídos dos artigos os seguintes dados: autores, ano da publicação, objetivo dos estudos, descrição da amostra, descrição da atuação do profissional na saúde mental, descrição do grupo controle (quando presente), principais resultados e conclusão do estudo. Os dados obtidos, após a leitura dos resumos e dos artigos, foram apresentados na forma de resumo descritivo em tabelas e em quadros.

\section{RESULTADOS E DISCUSSÃO}

O levantamento bibliográfico abrangeu publicações de janeiro de 2012 a janeiro de 2017 e, na busca inicial obteve 120 artigos, destes 113 foram excluídos pela análise de título e da leitura prévia do resumo, visto que não atenderam aos critérios estabelecidos de inclusão. Somente 8 artigos foram potencialmente relevantes e lidos na íntegra. O fluxo de estudos, por meio do processo de seleção dos artigos nas bases de dados, é apresentado na Tabela 1.

Tabela 1. Seleção dos artigos sobre a atuação do profissional em saúde mental nos Núcleos de Apoio à Saúde da Família.

\begin{tabular}{cccc}
\hline $\begin{array}{c}\text { Base de } \\
\text { Dados }\end{array}$ & $\begin{array}{c}\mathrm{N}^{\circ} \text { de Artigos } \\
\text { Encontrados }\end{array}$ & $\begin{array}{c}\mathrm{N}^{\circ} \text { de } \\
\text { Artigos } \\
\text { Excluídos }\end{array}$ & $\begin{array}{c}\mathrm{N}^{\circ} \text { de } \\
\text { artigos } \\
\text { Incluídos }\end{array}$ \\
\hline SciELO & 07 & 05 & 03 \\
LILACS & 52 & 47 & 05 \\
PubMed & 61 & 61 & 00 \\
\hline
\end{tabular}

Os artigos selecionados para leitura e análise descritos como: ano de publicação, autores, objetivo do trabalho, tipo de estudo, população e amostra, conforme o Quadro 1.

Quadro 1. Síntese dos artigos publicados nas bases de dados no período de janeiro de 2012 a janeiro de 2017.

\begin{tabular}{|c|c|c|c|c|}
\hline Ano & Autores & Objetivos & Tipo de estudo & População e amostra \\
\hline 2012 & $\begin{array}{c}\text { PINTO, A.G.A, et } \\
\text { al. }\end{array}$ & $\begin{array}{l}\text { Analisar a articulação das ações de } \\
\text { saúde mental entre as equipes da } \\
\text { Estratégia Saúde da Família e do } \\
\text { Centro de Atenção Psicossocial pelo } \\
\text { processo de matriciamento com ênfase } \\
\text { na integralidade do cuidado e } \\
\text { resolubilidade assistencial. }\end{array}$ & $\begin{array}{l}\text { Qualitativo numa } \\
\text { perspectiva crítica } \\
\text { e reflexiva }\end{array}$ & $\begin{array}{c}\text { Participaram } 32 \text { usuários } \\
\text { e } 22 \text { familiares atendidos } \\
\text { no matriciamento em } \\
\text { saúde mental, } 46 \\
\text { profissionais de saúde da } \\
\text { Estratégia Saúde da } \\
\text { Família e } 15 \text { dos Centros } \\
\text { de Atenção Psicossocial } \\
\text { de dois municípios do } \\
\text { Estado do Ceará. }\end{array}$ \\
\hline 2013 & $\begin{array}{l}\text { MINOZZO, F; } \\
\text { COSTA, I.I. }\end{array}$ & $\begin{array}{l}\text { Analisar a implantação do apoio } \\
\text { matricial entre CAPS III e equipes de } \\
\text { Saúde da Família. }\end{array}$ & $\begin{array}{l}\text { Qualitativo de } \\
\text { cunho } \\
\text { exploratório, }\end{array}$ & $\begin{array}{l}\text { Participaram 15 } \\
\text { profissionais do CAPS III } \\
\text { e } 6 \text { profissionais de nível } \\
\text { superior da Saúde da } \\
\text { Família. }\end{array}$ \\
\hline 2015 & HIRDES, A. & $\begin{array}{l}\text { Investigar o Apoio Matricial em saúde } \\
\text { mental na Atenção Primária à Saúde, } \\
\text { na perspectiva dos profissionais } \\
\text { generalistas, com vista à identificação } \\
\text { das diretrizes, princípios e valores } \\
\text { profissionais que permeiam o processo. }\end{array}$ & $\begin{array}{l}\text { Qualitativo } \\
\text { descritivo- } \\
\text { analítico }\end{array}$ & $\begin{array}{l}21 \text { participantes e } \\
\text { realizados três grupos } \\
\text { focais }(\mathrm{GF}) .\end{array}$ \\
\hline 2016 & $\begin{array}{l}\text { TATMATSU, } \\
\text { D.B.; ARAÚJO, } \\
\text { A.C.C. }\end{array}$ & $\begin{array}{l}\text { Analisar como os profissionais } \\
\text { inseridos nas atividades de Apoio } \\
\text { Matricial o têm avaliado, aludindo os } \\
\text { principais benefícios que tal estratégia } \\
\text { tem proporcionado às ações e serviços } \\
\text { de saúde de maneira geral. }\end{array}$ & $\begin{array}{l}\text { Qualitativo e } \\
\text { tipologia } \\
\text { descritiva }\end{array}$ & $\begin{array}{l}18 \text { entrevistas, entre } \\
\text { profissionais da atenção } \\
\text { primária e dos serviços } \\
\text { de saúde mental. }\end{array}$ \\
\hline 2012 & $\begin{array}{l}\text { VANNUCCHI, } \\
\text { A.M.C.; JUNIOR, } \\
\text { N.C. }\end{array}$ & $\begin{array}{l}\text { Analisar o processo de trabalho do } \\
\text { médico psiquiatra na Atenção Primária } \\
\text { de Saúde a partir das organizações } \\
\text { tecnológicas da UBS e do NAS F na } \\
\text { cidade de São Paulo. }\end{array}$ & Qualitativo & $\begin{array}{l}\text { Os sujeitos da pesquisa } \\
\text { foram dois médicos } \\
\text { psiquiatras. }\end{array}$ \\
\hline 2015 & MARTINS, & Conhecer os procedimentos, as ações e & Qualitativo & Participaram do estudo \\
\hline
\end{tabular}




\begin{tabular}{|c|c|c|c|c|}
\hline & A.K.L. et al. & $\begin{array}{l}\text { condutas adotadas em saúde mental no } \\
\text { âmbito da atenção básica. }\end{array}$ & exploratória & $\begin{array}{l}22 \text { sujeitos, destes, } \\
\text { médicos, enfermeiras, } \\
\text { técnicos de enfermagem, } \\
\text { agentes administrativos, } \\
\text { agentes comunitários de } \\
\text { saúde e acadêmicos de } \\
\text { enfermagem e medicina } \\
\text { envolvidos nas práticas } \\
\text { do serviço. }\end{array}$ \\
\hline 2012 & $\begin{array}{l}\text { RODRIGUES, } \\
\text { E.S.; MOREIRA, } \\
\text { I. B. }\end{array}$ & $\begin{array}{l}\text { Analisar o processo de interlocução da } \\
\text { saúde mental com a atenção básica, em } \\
\text { curso no município de Vitória-ES. }\end{array}$ & Qualitativa & $\begin{array}{c}14 \text { entrevistas, } 6 \\
\text { ocorreram com profissio- } \\
\text { nais da UBS da Família } \\
\text { de Santo André e } 8 \text { com } \\
\text { profissionais de } \\
\text { diferentes regiões de } \\
\text { saúde do CAPS da Ilha } \\
\text { de Santa Maria }\end{array}$ \\
\hline 2012 & $\begin{array}{l}\text { AZEVEDO, I.M.; } \\
\text { SANTOS, A.T. }\end{array}$ & $\begin{array}{c}\text { Identificar o conhecimento dos } \\
\text { Enfermeiros da ESF sobre a assistência } \\
\text { em saúde mental a partir da RP; e } \\
\text { investigar a articulação entre CAPS e } \\
\text { ESF no tocante às atividades de } \\
\text { matriciamento. }\end{array}$ & $\begin{array}{l}\text { Qualitativo } \\
\text { descritivo }\end{array}$ & $\begin{array}{l}8 \text { enfermeiros entrevistos } \\
\text { da ESF de Parelhas-RN. }\end{array}$ \\
\hline
\end{tabular}

Quanto ao desenho metodológico, os estudos tiveram predominância com abordagens qualitativa, descritiva e exploratória. O idioma de publicação predominante foi o português.

Nesse aspecto, evidenciou-se que os artigos encontrados, em sua maioria, foram desenvolvidos por equipe multiprofissional, seguidos por estudos realizados por médicos e enfermeiros.

A discussão dos resultados foi definida de acordo com a análise descritiva entre os artigos, que possibilitaram o entendimento da atuação dos profissionais na saúde mental nos NASFs em dois eixos temáticos: apoio matricial em saúde mental na Estratégia Saúde da Família (ESF) e atuação do profissional na saúde mental.

\section{Apoio matricial em saúde mental na ESF}

O matriciamento em saúde mental caracteriza-se como um pilar ofertado às equipes multiprofissionais da atenção primária em saúde, com o intento de organização e de ampliação das ações à saúde no Sistema Único de Saúde (SUS). Nesse sentido, observou-se, nos artigos selecionados, a importância da integração do campo psicossocial no processo operacional da ESF e, com isso, fica evidente não apenas o cuidado em saúde mental na assistência direta das equipes de saúde com a participação dos profissionais, mas também a participação dos familiares.

Segundo o estudo de Pinto et al. (2012), a implantação do apoio matricial em saúde mental, além de ter sido um foco intersetorial, promoveu uma atenção integral no campo psicossocial com vínculos entre o Centro de Atenção Psicossocial (CAPS) e as unidades de Saúde da Família.

No cenário hodierno do Brasil, ainda há profissionais da ESF com dificuldades na abordagem aos pacientes que apresenta distúrbio mental por falta de capacitação e elegem pensamentos biomédicos e hospitalocêntricos (MINOZZO; COSTA, 2013).
Em contrapartida a essa perspectiva, no estudo de Figueiredo e Campos (2009) observou-se nos relatos dos profissionais, a percepção de que o apoio matricial é uma forma de capacitação e troca de experiências, o que ocasiona uma estratégia valorizada, criada para orientar e dar suporte aos atendimentos.

Dessa forma, a saúde mental é, ainda, uma área que necessita ser fortalecida na atenção primária, já que o apoio matricial exige habilidades por partes dos profissionais que possam subsidiar gestores e equipes na efetividade das práticas de assistência psicossocial.

O processo de matriciamento no sistema de saúde é composto por uma equipe de referência que atuam na ESF e uma equipe de apoio matricial, formada por um grupo interdisciplinar da saúde que, nesse caso, seria em saúde mental. Essa relação entre equipes tem como finalidade propor arranjos organizacionais e desenvolver uma metodologia para administração do trabalho em saúde (BRASIL, 2011).

Nesse sentido, é notório uma fragilidade que merece ser discutida quanto à efetividade de políticas públicas voltadas à integralidade do cuidado. $\mathrm{O}$ envolvimento das equipes da ESF e a troca de experiências aparecem como facilitadores para a execução do apoio matricial a fim de minimizar os modelos privatistas e poder direcionar o atendimento ao CAPS (HIRDES, 2015). Com isso, é fundamental o processo de humanização, a motivação para o atendimento e o vínculo centralizado na pessoa e não apenas na doença.

O matriciamento em saúde mental na atenção primária envolve, sobretudo, a articulação do CAPS com as equipes de Saúde da Família e, a partir dessa prática, objetiva-se diminuir o direcionamento para esses centros psicossociais, em função da presença dos especialistas na área de cobertura da ESF durante a resolutividade dos casos propostos (PEGORARO; CASSIMIRO; LEÃO, 2014).

Para Tatmatsu e Araújo (2016), um dos benefícios da execução do apoio matricial para os profissionais nos 
centros de Saúde da Família do município de Fortaleza, Ceará, foi a comoção da equipe em relação ao sofrimento psíquico, a importância do trabalho interdisciplinar e a organização do fluxo de demanda. Isso é uma importante ferramenta da reforma psiquiátrica no contexto atual.

Nesse sentido, é de grande valia o encontro de profissionais de distintas áreas na execução do Projeto Terapêutico Singular (PTS) a fim de construir uma compreensão integral do processo saúde e doença baseando-se nos princípios doutrinários do SUS.

\section{Atuação do profissional na saúde mental na atenção básica}

A ESF preconiza, por ser um projeto do SUS, a universalidade, a equidade e a integralidade no cuidado. Por isso, surgiu a implantação de ações voltadas à saúde mental na Atenção Básica. Nesse contexto, o estudo de Martins et al. (2015) questionou alguns profissionais da ESF, no município de Juazeiro do Norte-CE, que a atuação na saúde mental ainda é biologicista e que muitos profissionais desconhecem a função da rede de atenção à saúde mental e isso gera complicações na assistência nos diferentes níveis, o que rompe a continuidade do acesso e a qualidade do serviço.

A descentralização do cuidado, assim como um trabalho interdisciplinar são potencialidades decorrentes do benefício de uma assistência mais dinâmica através da efetividade das redes psicossociais. Embora seja um desafio traçar novas possibilidades de recuperação e reinserção desses usuários num exercício de reconhecimento de sua cidadania (COSTA et al., 2012).

Ademais, a realização de trabalhos com os usuários e os familiares é fundamental para quebrar as barreiras da equipe da ESF e os CAPS, para que, assim, possam trabalhar juntos no treinamento dos profissionais da atenção primária com foco na atuação entre os pacientes e familiares. Dessa forma, os profissionais passam a ser os condutores do processo saúde e não apenas expectadores da doença (AZEVEDO; SANTOS, 2012).

Segundo o estudo de Vannucchi e Junior (2012), a inserção do psiquiatra, focalizado no NASF, não prevê a necessidade de assistência psiquiátrica baseada no modelo hospitalocêntrico. Isso acaba por interferir no apoio matricial e na resolubilidade dos problemas da atenção primária de saúde. Em resposta a esse estudo, percebeu-se que ainda existe grandes obstáculos, dentre os quais podem ser destacados a falta de confiança e o desconhecimento, por parte do profissional psiquiatra, da importância de um olhar mais integral aos usuários portadores de transtorno mental, já que ficou evidente na literatura analisada a necessidade de desconstrução de práticas fundamentadas na institucionalização, sendo necessário o enfoque no sujeito, como um ser complexo..

Para que haja um melhor envolvimento dos profissionais no contexto comunitário de maneira integral, regido por princípios éticos antimanicomiais, é preciso uma atuação na educação permanente de todos os profissionais que atuam tanto nas ESF quanto nos NASFs, além da necessidade de articulação dos serviços da rede de atenção à saúde.

O trabalho de Rodrigues e Moreira (2012) realizado no município de Vitória, Espírito Santo, no CAPS e na Unidade Básica de Saúde (UBS), reforçou a ideia de insegurança apontada anteriormente pois, através dos relatos dos trabalhadores, ainda há insegurança quanto ao cuidado em saúde mental e encaminham os pacientes aos Centros de Atenção Psicossocial (CAPS), por ser um ponto de atenção à saúde.

Embora cada profissional apresente seu processo de trabalho particular, é de suma importância que os profissionais da Equipe de Saúde da Família não só se integrem entre si, mas também com a comunidade que atua, na busca de uma melhor interpretação acerca da realidade social e, com isso, poder desenvolver ações mais efetivas na perspectiva de proporcionar uma melhor qualidade de vida e emocional de seus pacientes (CAMPOS JÚNIOR; AMARANTE, 2015).

Em uma pesquisa realizada em Uberlândia, concluiuse que ainda há uma grande lacuna entre as diretrizes propostas pela política de saúde mental e as práticas realizadas pelos profissionais, sobretudo na atenção básica, pois ainda há uma resistência na superação do modelo biomédico e dos hospitais psiquiátricos (SILVA, 2014).

Assim, nem sempre o serviço de atenção primária dispõe de condições para ofertar suporte a essa demanda de atendimento voltado para pacientes com transtorno mental, como a falta de recursos e capacitação profissional, que acaba por prejudicar o desenvolvimento de uma interação na equipe multiprofissional (GAZIGNATO; SILVA, 2014).

Um estudo realizado em duas UBSs em Osasco - SP percebeu que os serviços realizados pelas equipes de ESF e dos NASFs não são suficientes para assegurar a integralidade do cuidado, em especial a saúde dos sujeitos com transtorno mental grave (BERTAGNONI et al., 2012).

Dessa forma, demarca-se aqui a necessidade de estudos que envolvam não apenas o efeito da atuação dos profissionais de saúde mental na atenção básica, como também as dificuldades e as potencialidades de atendimento, a fim de se desenvolver uma prática efetiva no cuidado ao paciente com sofrimento psíquico.

\section{CONCLUSÕES}

A atenção básica é um espaço privilegiado para as ações de saúde mental, haja vista que, pela aproximação com a comunidade, é possível resolver os problemas base, proporcionando não só o acompanhamento efetivo, como também na reinserção desses usuários no convívio social.

É fundamental a adoção do apoio matricial as equipes multiprofissionais, com o intento de ampliar as ações de saúde, fomentar a humanização da assistência e aperfeiçoar a atuação do profissional de saúde mental nos NASFs de forma não apenas direcionada, mas também holística.

\section{REFERÊNCIAS}

AZEVEDO, D. M.; SANTOS, A. T. Ações de saúde mental na atenção básica: conhecimento de enfermeiros sobre a reforma psiquiátrica. Revista de Pesquisa: Cuidado é Fundamental Online, v. 4, n. 4, p. 3006-14, 2012.

BERTAGNONI, L.; MARQUES, A. L. M.; MURAMOTO, M. T.; MÂNGIA, E. F. Núcleo de Apoio 
à Saúde da Família e Saúde Mental: itinerários terapêuticos de usuários acompanhados em duas Unidades Básicas de Saúde. Revista de Terapia Ocupacional da Universidade de São Paulo, v. 23, n. 2, p. 153-162, 2012.

BRASIL. Ministério da Saúde. Reforma Psiquiátrica e política de saúde mental no Brasil. Relatório Regional de Reforma dos Serviços de Saúde Mental: 15 anos depois de Caracas. Brasília, 2005.

BRASIL. Ministério da Saúde. Gabinete do Ministro Portaria $n^{\circ} 154$ Cria os Núcleos de Apoio à Saúde da Família - NASF. Brasília, 2008

BRASIL. Ministério da Saúde. Guia prático de matriciamento em saúde mental. Ministério da Saúde: Centro de Estudo e Pesquisa em Saúde Coletiva, 2011.

BRASIL. Ministério da Saúde. Guia de Saúde Mental: atendimento e intervenção com usuários de álcool e outras drogas. Brasília, 2013.

BRASIL. Secretaria de Atenção à Saúde. Departamento de Atenção Básica. Saúde mental / Departamento de Ações Programáticas Estratégicas. Brasília, p. 176, 2013.

BRASIL. Ministério da Saúde. Cadernos de Atenção Básica no 34 - Saúde Mental. Brasília, Editora MS, 2013, $1^{\mathrm{a}}$ ed.

BRASIL. Ministério da Saúde. Saúde Mental em Dados 12, ano 10, $\mathrm{n}^{\mathrm{o}}$ 12. Informativo eletrônico. Brasília, 2015. Disponível

em: <http://www.mhinnovation.net/sites/default/files/downloa ds/innovation/reports/Report_12-edicao-do-SaudeMental-em-Dados.pdf>. Acesso em: 09 out 2017.

CAMPOS JUNIOR, A.; AMARANTE, P. D C. Estudo sobre práticas de cuidado em saúde mental na Atenção Primária: o caso de um município do interior do estado do Rio de Janeiro. Cadernos Saúde Coletiva, v. 23, n. 4, p. 425-435, 2015.

CORREIA, V. R.; BARROS, S.; COLVERO, L. A. Saúde mental na atenção básica: prática da equipe de saúde da família. Revista da Escola de Enfermagem da USP, São Paulo, v. 45, n. 6, p. 1501-6, 2011.

COSTA, A.; SILVEIRA, M.; VIANNA, P.; SILVAKURIMOTO, T. Desafios da Atenção Psicossocial na Rede de Cuidados do Sistema Único de Saúde do Brasil. Revista Portuguesa de Enfermagem de Saúde Mental, n. 7, p. $46-53,2012$.

DE SOUZA, M. T.; DA SILVA, M. D.; DE CARVALHO, R. Revisão integrativa: o que é e como fazer. Einstein, v. 8, n. 1 Pt 1, p. 102-6, 2010.

ERVIN, D. A.; WILLIAMS, A.; MERRICK, J. Primary care: mental and behavioral health and persons with intellectual and developmental disabilities. Frontiers in public health, v. 2, 2014.
FIGUEIREDO, M. D.; CAMPOS, R. O. Saúde Mental na atenção básica à saúde de Campinas, SP: uma rede ou um emaranhado? Ciência \& Saúde Coletiva, v. 14, n. 1, p. 129-138, 2009.

FIGUEIREDO, M. D.; CAMPOS, R. O. Saúde Mental e Atenção Básica à Saúde: o apoio matricial na construção de uma rede multicêntrica. Saúde em Debate, v. 32, n. 7879-80, p. 143-149, 2008.

GAZIGNATO, E. C. S.; SILVA, C. R. C. Saúde mental na atenção básica: o trabalho em rede e o matriciamento em saúde mental na Estratégia de Saúde da Família. Saúde em Debate, v. 38, p. 296-304, 2014.

HIRDES, A. A perspectiva dos profissionais da Atenção Primária à Saúde sobre o apoio matricial em saúde mental. Revista Ciência \& Saúde Coletiva, v. 20, n. 2, p. 371-382, 2015.

KAKUSHI, L. E.; ÉVORA, Y. D. M. As redes sociais na educação em enfermagem: revisão integrativa da literatura. Revista Latino-Americana de Enfermagem, v, 24, p. 2709, 2016.

MARTINS, A. K. L.; SOUZA, A. M. A.; VIEIRA, N. F. C.; PINHEIRO, P. N. D. C.; BRAGA, V. A. B. Práticas em saúde mental na estratégia saúde da família: um estudo exploratório. Revista de Pesquisa: Cuidado é Fundamental Online, v. 7, n. 1, p. 1905-14, 2015.

MINOZZO, F.; COSTA, I. I. Apoio matricial em saúde mental entre CAPS e Saúde da Família: trilhando caminhos possíveis. Psico-USF, Bragança Paulista, v. 18, n. 1 , p. 151-160, 2013.

OMS. Organização Mundial da Saúde. Integração da saúde mental nos cuidados de saúde primários: Uma perspectiva global. Brasília, 2007. Disponível em: <http://www.who.int/eportuguese/publications/pt/>.

Acesso em: 09 out 2017.

ORUCHE, U. M.; DRAUCKER, C. B.; KHATTAB, H. et al. The Challenges for Primary Caregivers of Adolescents with Disruptive Behavior Disorders. Journal of Family Nursing, v. 21, n. 1, p. 149-167, 2015.

PINTO, A. G. A.; JORGE, M. S. B.; VASCONCELOS, M. G. F.; SAMPAIO, J. J. C.; LIMA, G. P.; BASTOS, V. C.; SAMPAIO, H. A. D. C. Apoio matricial como dispositivo do cuidado em saúde mental na atenção primária: olhares múltiplos e dispositivos para resolubilidade. Revista Ciência \& Saúde Coletiva, v. 17, n. 3 , p. $653-660,2012$

PEGORARO, R. F.; CASSIMIRO, T. J. L.; LEÃO, N. C. Matriciamento em saúde mental segundo profissionais da Estratégia da Saúde da Família. Psicologia em Estudo, v. 19, n. 4, 2014.

RODRIGUES, E. S.; MOREIRA, M. I. B. A Interlocução da Saúde Mental com Atenção Básica no Município de 
Vitoria/ES. Saude e Sociedade, v. 21, n. 3, p. 599-611, matricial. Revista Mudanças - Psicologia da Saúde, v. 24, 2012. n. 2, p. 71-79, 2016.

SCHUtel, T. A. A.; ROdRIGUES, J.; PERES, G. M. A VANnUCCHI, A. M. C.; JUNIOR, N. C. Modelos concepção de demanda em saúde mental na Atenção tecnoassistenciais e atuação do psiquiatra no campo da Primária à Saúde. Repositório Institucional da atenção primária à saúde no contexto atual do Sistema Universidade Federal de Santa Catarina, v. 8, n. 2, p. 95- Único de Saúde, Brasil. Physis: Revista de Saúde 93, 2015.

Coletiva, v. 22, n. 3, p. 963-982, 2012.

SILVA, L. M. B. Projeto de intervenção em saúde mental da UBSF de Tapuirama. Uberaba: UFTM, 2014.

WHO. World Heath Organization. Mental disorders. Genebra, 2015. Disponível em:

TATMATSU, D. B.; ARAÚJO, A.C.C. Atenção primária <http://www.who.int/mediacentre/factsheets/fs396/en/>.

e saúde mental: contribuições e potencialidades do apoio Acesso em: 08 out 2017. 